\title{
Ultrasonographic assessment of the cutaneous changes induced by topical flavonoid therapy
}

This article was published in the following Dove Press journal:

Clinical, Cosmetic and Investigational Dermatology

9 January 2012

Number of times this article has been viewed

\author{
Diana Crisan' \\ Maria Crisan ${ }^{2}$ \\ Mirela Moldovan ${ }^{3}$ \\ Monica Lupsor ${ }^{4}$ \\ Radu Badea ${ }^{4}$ \\ 'Student, Faculty of Medicine, \\ ${ }^{2}$ Department of Histology, \\ ${ }^{3}$ Department of Dermopharmacy \\ and Cosmetics, ${ }^{4}$ Department \\ of Ultrasonography, University \\ of Medicine and Pharmacy "Iuliu \\ Hatieganu” Cluj-Napoca, Romania
}

Correspondence: Maria Crisan Department of Histology/Dermatology, UMPh luliu Hatieganu, Cluj-Napoca,

Romania

Tel +40 744766496

Email maria.crisan@umfcluj.ro

\begin{abstract}
Ultrasonography allows the quantification of dermal density and echogenicity changes during the physiological senescence process. Some active ingredients are able to slow down the tissular degeneration and disorganization process. The purpose of this study was to assess the cutaneous changes induced by the topical use of products containing Viniferol ${ }^{\circledR}$ as active ingredient, using high-frequency ultrasound. The study was performed over 12 weeks and included 80 healthy Caucasian female subjects, aged 22-75 years, divided into two groups: the study group and the control group. The product was applied according to a predetermined protocol. The measurements performed for each subject were: the thickness of the epidermis and dermis ( $\mathrm{mm})$, the number of low, medium, and high echogenic pixels, and the number of low echogenic pixels in the upper dermis/number of low echogenic pixels in the lower dermis. All the parameters showed a significant improvement. Ultrasound measurements showed an increase of the mean thickness of the epidermis $(P<0.0001)$ and dermis $(P<0.0001)$ following the application of the Viniferol product as compared to the control group. The changes in the dermal echogenicity confirm the efficacy and direct action of Viniferol upon the cutaneous fibroblasts. No side effects related to the treatment were recorded. The study proves the efficacy of this active ingredient in the cutaneous senescence process as well, as the fact that anti-aging prophylaxis should be initiated in the 20-40 year critical age group. This interval involves specific changes in dermal echogenicity that quantify intense molecular, biochemical and structural changes, being thus mostly and highly responsive to the anti-aging therapy.
\end{abstract}

Keywords: ultrasonography, flavonoid therapy, cutaneous changes, anti-aging therapy

\section{Introduction}

High-frequency ultrasound is a noninvasive diagnostic method with several applications in the field of internal medicine and, recently, clinical dermatology as well as dermatooncology and dermatocosmetics. ${ }^{1,2}$ It can represent a "noninvasive histological tool" for the quantification of the cutaneous structure or of the cutaneous pathology by monitoring the evolution of cutaneous lesions and the assessment of topical therapies' efficacy in chronic inflammatory disorders (morphea, scleroderma, psoriasis), for the quantification of the intrinsic and extrinsic senescence process, as well as the efficacy of topical or generally applied therapies. ${ }^{3}$ It can also be used as an important research tool of the cutaneous senescence process and the efficacy of various anti-aging therapies. ${ }^{4,5}$

The use of high-frequency ultrasound in the assessment of the regenerative effects of various anti-aging therapies is an exciting and important area for research. From all the available imaging methods, high-frequency ultrasound (Dermascan $20 \mathrm{MHz}$ Cortex Technology) is a new study tool focused on the assessment of the cutaneous 
physiological and pathological features, having a penetration depth of up to $2.5 \mathrm{~cm}$.

Collagen is an echogenic marker protein, synthesized by dermal fibroblasts, which can be identified by high-frequency ultrasound. The changes of the extracellular matrix, consisting in variations of the dermal density and echogenicity throughout the physiological senescence process, or during several anti-aging therapies quantify subtle reactions at molecular, cellular, and tissular level that influence the local homeostasis. ${ }^{6}$

High-frequency ultrasound allows an objective assessment of the cutaneous structure: the thickness of the epidermis and dermis as well as the dermal density, which are useful parameters for the quantification of the cutaneous regeneration process. ${ }^{7,8}$ Several changes of the cutaneous structure after topical prednisone therapy in children, or anti-aging natural supplement therapies in adults were ultrasonographically successfully assessed. ${ }^{9}$

Previous studies have shown that the thickness of the epidermis and dermis, as well as the dermal density are important parameters that assess the cutaneous regeneration process.

The neosynthesis of the protein structures induces an increase of the dermal echogenicity and density, local cell architecture changes, and an increase of the dermis and epidermis thickness. The epidermis is the morphological expression of the changes in the subjacent dermis. ${ }^{7}$

It has been proved that certain ultrasonographic markers, such as SLEB (subepidermal low echogenity band) or the LEPs/LEPi ratio (number of low echogenic pixels in the upper dermis/number of low echogenic pixels in the lower dermis) can quantify the cutaneous senescence process, as well as the efficacy of various anti-aging therapies. ${ }^{2,10,11}$

As far as the anti-aging therapy is concerned, Viniferol, a standardized extract from Bordeaux vine stalks, is one of the newest and very efficient anti-wrinkle and anti-aging agents. The active ingredient of Viniferol is $\varepsilon$-viniferine, a generic name for a new class of phytoalexins characterized as resveratrol dimers. Besides the wellknown antioxidant effect of this flavonoide-containing extract, it has a direct action on the protein expression genes involved in the proliferation and differentiation of integumentary cells. These are retinoid-like effects which decrease cellular differentiation and increase cellular growth. Due to this action, Viniferol profoundly restructures and regenerates the skin and due to its antioxidant properties, it reestablishes the metabolic balance of the cutaneous cells, slowing down the tissular degeneration and disorganization process. ${ }^{12}$
Even though the general anti-aging effects of the flavonoids are well known, until now no scientific studies investigated the action of Viniferol at the cutaneous level by using high-frequency ultrasound.

The purpose of the study is the assessment, with the help of high-frequency ultrasound, of the cutaneous changes induced by topical use of products containing Viniferol.

\section{Materials and methods Patients}

Eighty female subjects, aged $22-75$ years, who requested prophylaxis and anti-aging therapy with flavonoids, were prospectively included in the study. Half $(50 \%)$ of the subjects belonged to Fitzpatrick phototype class II and 50\% to phototype class III. ${ }^{13}$ The study excluded patients with known allergies to topical flavonoids, cutaneous facial lesions, resurfacing or other anti-aging therapies in the last 2 months, or those who used phototherapy or oral contraception.

The subjects taken into the study were divided into two categories: a study group and a control group. The study group followed the proposed anti-aging therapy for 12 weeks, according to a standard protocol. In the morning, a hydrating emollient cream, based on occlusive hydrating agents was applied at the facial level (including the zygomatic area) by lightly massaging the area for 2 minutes. ${ }^{14}$ In the evening, an anti-aging product containing Viniferol, was applied in the same manner. No other cosmetic products were used by the subjects during the 12 weeks of study. The control group followed a placebo therapy for 12 weeks, using only moisturizing cream in the morning and evening, applied at the facial level, including the zygomatic area.

For every subject, ultrasonographic images were taken from the zygomatic level at the beginning and 12 weeks after the local application of the emollient, hydrating product, and the anti-aging, Viniferol-based cream.

The study was approved by the Ethical Committee of University of Medicine and Pharmacy Iuliu Hatieganu, Cluj-Napoca, Romania. Every subject was informed about the nature and purpose of the study, and signed a written consent before enrolling into the study.

\section{Ultrasonographic evaluation}

The ultrasonographic evaluation was performed with the Dermascan equipment (Cortex Technologies, Hadsund, Denmark), containing a $20 \mathrm{MHz}$ transducer, that allows the "in vivo" acquirement of sectional cutaneous images up to a depth of $2.5 \mathrm{~cm}$.

Dermascan consists of three major components: a transducer, an elaboration system, and a database (Figure 1). 


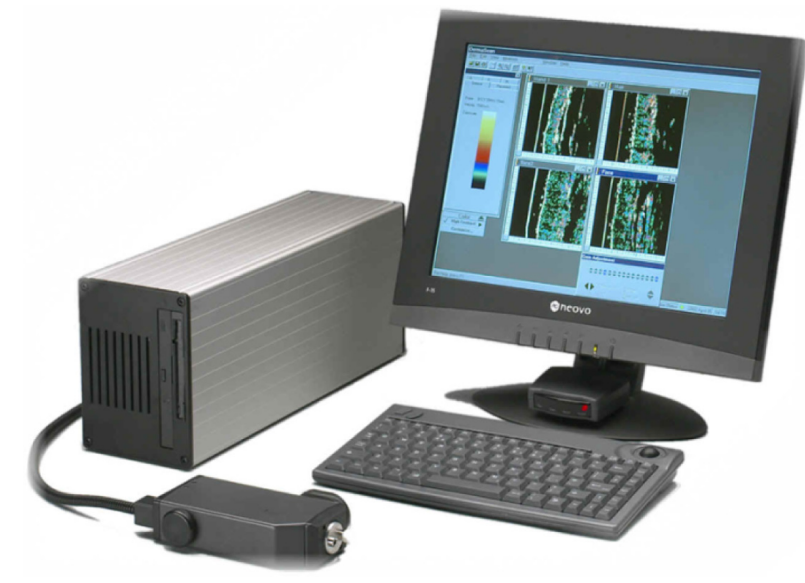

Figure I Ultrasonographic equipment (Dermascan C, Cortex Technology).

The ultrasonic wave is partially reflected at the boundary between adjacent structures and generates echoes of different amplitudes. The intensity of the reflected echoes is evaluated by a microprocessor and visualized as a colored two-dimensional image. ${ }^{1}$ The color scale of echogenicity is: white-yellow - redgreen - blue - black. Normally, the epidermal echogenicity appears as a white band, the dermis is expressed as a two-color composition: yellow and/or red, and the subcutaneous layer appears either green or black (Figure 2).

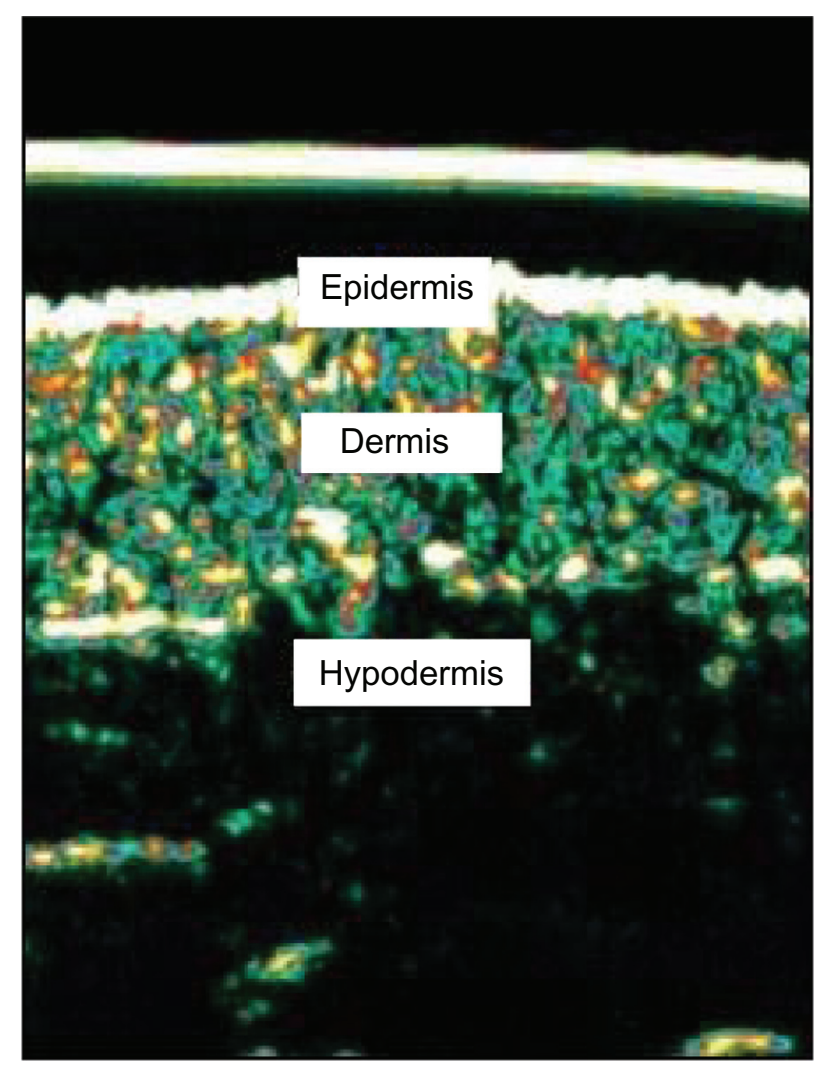

Figure 2 Ultrasound image of the skin: epidermis, dermis, hypodermis.
The transducer was applied on the integument, on the left zygomatic area, parallel to the zygomatic arcade, after previously having cleaned the cutaneous surface and applied ultrasonographic gel. The gain curve was adjusted at a value of $20 \mathrm{~dB}$, at a speed of ultrasound at tissue level of $1580 \mathrm{~m} / \mathrm{s}$.

The obtained images were further processed with image analysis software (Dermavision, Cortex Technology).

For every subject, the following parameters were measured on the images obtained before and after therapy: thickness of the epidermis and dermis (mm), the number of LEP (low echogenic pixels), MEP (medium echogenic pixels), HEP (high echogenic pixels), LEPs/LEPi ratio.

The thickness of the epidermis was obtained by calculating the mean of three measurements performed at three different sites of each image (the two extremities of the analyzed image and the center of the image). The thickness of the dermis was obtained by measuring the distance between the dermo-epidermal and the dermo-hypodermic junction at the same three different sites and by establishing the mean of the three values.

The analysis software used has the pixel amplitude corresponding to a numerical scale set between 0-255. By selecting a certain interval from the $0-255$ scale, we obtained values corresponding to a certain pixel type, present in the analyzed image. Thus, the 0-30 interval corresponds to LEP, the 50-150 interval to MEP, and the 200-255 interval to HEP.

The number of LEP were determined separately in the upper (LEPu) and lower (LEPl) dermis. The boundary between these regions was the line chosen as parallel to the epidermal entrance echo and dividing the dermal image into two parts of equal thickness. The ratio of LEP number in the upper and lower dermis (LEPs/LEPi) was calculated.

\section{Statistical analysis}

Statistical analysis was performed with SPSS (v 15.0; SPSS Inc, Chicago, IL). The data we obtained were analyzed, calculating the mean and standard deviation for the quantitative variables of every group and the proportions for the qualitative variables. The difference of means before and after treatment was tested using a $t$-test for paired samples, and the relationship between different parameters was assessed through Spearman's correlation coefficients. A $P$ value $<0.05$ was considered significant.

\section{Results and discussion}

Forty female subjects with a mean age of $49.60 \pm 14.19$ years (range 22-75 years) were included in the study group. Twelve subjects (30\%) were under the age of 40,16 subjects 
(40\%) between 40-60 years and twelve (30\%) over the age of 60 . The control group was composed of subjects aged $22-75$ years as well, divided into the above mentioned age categories.

All subjects involved in the study tolerated the therapy, without evoking adverse effects (erythema, pruritus, ocular disturbance). Subjectively, post-flavonoid therapy, a significant hydration of the skin throughout the day and an increase of the cutaneous tonicity was noticed.

The thickness of the epidermis in the study group initially was $0.129 \pm 0.237 \mathrm{~mm}$, and the thickness of the dermis $1.434 \pm 0.241 \mathrm{~mm}$.

After therapy, an increase of the mean thickness of the epidermis $(0.129 \pm 0.237 \mathrm{~mm}$ vs $0.150 \pm 0.323 \mathrm{~mm}, P<0.000)$, and of the dermis $(1.434 \pm 0.241 \mathrm{~mm}$ vs $1.569 \pm 0.219 \mathrm{~mm}$, $P<0.0001$ ) was observed (Tables 1 and 2).

The thickness of the dermis increased mainly in the 40-60 years age group $(1.413 \pm 0.280 \mathrm{~mm}$ vs $1.569 \pm 0.279 \mathrm{~mm}$, $P=0.001)$, and less, but still significantly in the $<40$ years group $(1.416 \pm 0.266 \mathrm{~mm}$ vs $1.585 \pm 0.150 \mathrm{~mm}, P=0.015)$, while in those $>60$ years, the increase was not statistically significant $(1.480 \pm 0.157 \mathrm{~mm}$ vs $1.554 \pm 0.204 \mathrm{~mm}$, $P=0.097)$.

At the same time, at the dermal level, the number of LEP decreased $(15,153.53 \pm 3589.86$ vs $12,958.48 \pm 3628.35$, $P<0.0001)$, but this aspect was only noticed in the lower dermis $(6949.75 \pm 1966.93$ vs $6257.62 \pm 2224.88$, $P=0.016)$, not in the upper dermis $(7290.55 \pm 1794.60 \mathrm{vs}$ $6940.65 \pm 2150.30, P=0.168)$.

Overall, the LEPs/LEPi ratio increased significantly after flavonoid therapy $(1.092 \pm 0.330$ vs $1.259 \pm 0.631$, $P=0.011)$.

We also noticed an increase of MEP $(3359.72 \pm 1457.36 \mathrm{vs}$ $3983.47 \pm 1401.24, P=0.013)$ and $\operatorname{HEP}(460.27 \pm 323.93 \mathrm{vs}$ $750.90 \pm 493.82, P<0.0001)$ after therapy.

Table I Cutaneous parameters quantified by high-frequency ultrasound before and after treatment

\begin{tabular}{lccl}
\hline & Before treatment & After treatment & $\boldsymbol{P}$ value \\
\hline $\begin{array}{l}\text { Thickness of } \\
\text { epidermis }(\mathrm{mm})\end{array}$ & $0.13 \pm 0.24$ & $0.15 \pm 0.32$ & $<0.000 \mathrm{I}$ \\
$\begin{array}{l}\text { Thickness of } \\
\text { dermis }(\mathrm{mm})\end{array}$ & $1.43 \pm 0.24$ & $1.57 \pm 0.22$ & $<0.000 \mathrm{I}$ \\
LEP & & & \\
MEP & $15,153.53 \pm 3589.86$ & $12,958.48 \pm 3628.35$ & $<0.000 \mathrm{I}$ \\
HEP & $3359.72 \pm 1457.36$ & $3983.47 \pm 1401.24$ & 0.013 \\
LEPs & $460.27 \pm 323.93$ & $750.90 \pm 493.82$ & $<0.000 \mathrm{I}$ \\
LEPi & $7290.55 \pm 1794.60$ & $6940.65 \pm 2150.30$ & 0.168 \\
LEPs/LEPi & $6949.75 \pm 1966.93$ & $6257.62 \pm 2224.88$ & 0.016 \\
\hline
\end{tabular}

The general variation pattern of the quantifiable ultrasonographic parameters after flavonoid therapy is illustrated in Table 1 and Figure 3.

If we consider the variation of the ultrasonographic parameters after topical flavonoid therapy according to the phototype class of the subjects, it can be noticed that after therapy, there is a significant increase of the LEPs/LEPi ratio in the subjects belonging to phototype class II, not III (Table 2).

In the placebo group, we noticed no significant thickening of the epidermis and a slight increase of the dermis after therapy $(1.433 \pm 0.34 \mathrm{~mm}$ vs $1.486 \pm 0.14 \mathrm{~mm})$. The number of LEP at dermal level also show a slight increase $(13,213 \pm 1284$ vs $15,374 \pm 2318, P=0.1)$ due to an optimal hydration of the skin and a discrete decrease of high echogenity pixels $(421.8 \pm 121.18$ vs $368.3 \pm 104.03, P=0.07)$. The LEPs/LEPi ratio showed no particular display according to the age or phototype of the subjects.

The obtained results are in accordance with the data published in literature. Thus, locally applied flavonoids induce the neosynthesis of the fibrillary structures, but also of glycosaminoglycans, which display intense hydrophilic properties, favoring the cutaneous hydration.

It is well known that flavonoids have important anti-aging properties not only at a cutaneous level, but at the level of the entire organism. Viniferol, a molecule with proven antiaging and antioxidant properties, exhibits a complex action at the cutaneous level: it interacts with fibroblastic receptors, amplifies the interrelation fibrocyte-extracellular matrix, modulates the adhesiveness molecules and interferes with the oxidative stress process and nonenzymatic glycation, with a regenerative effect at cutaneous level. ${ }^{15}$

After therapy, a significant increase of the mean thickness of the epidermis and dermis was noticed, which once again confirms the presence of a complex, regenerative dermal process, induced by flavonoids. ${ }^{16,17}$

The dermal thickness increased the most in the 40-60 years age group, to a lesser extent, but still significantly under the age of 40 years, and insignificantly over 60 years. We can affirm that topical flavonoid products have the best efficacy on mature integument, with specific structural and hormonal characteristics. In young subjects ( $<40$ years) the thickness of the dermis increases discretely as the dermis is a young connective tissue, rich in glycosaminoglycans and thus, properly hydrated. ${ }^{18}$

After the age of 60, the extracellular matrix (fibrocytes, fibers, glycosaminoglycans) of the skin undergoes characteristic degenerative changes. Therefore, the flavonoid-based anti- 
Table 2 Variation of the cutaneous parameters quantified by high-frequency ultrasound before and after treatment, according to phototype

\begin{tabular}{|c|c|c|c|c|c|c|}
\hline & \multicolumn{3}{|l|}{ Phototype III } & \multicolumn{3}{|l|}{ Phototype II } \\
\hline & Before treatment & After treatment & $P$ value & Before treatment & After treatment & $P$ value \\
\hline Epidermis (mm) & $0.1288 \pm 0.026$ & $0.151 \pm 0.027$ & $<0.0001$ & $0.129 \pm 0.021$ & $0.148 \pm 0.037$ & 0.020 \\
\hline Dermis (mm) & $I .44 I \pm 0.270$ & $1.570 \pm 0.263$ & 0.001 & $\mathrm{I} .427 \pm 0.214$ & $1.568 \pm 0.172$ & 0.003 \\
\hline LEP & $15,059.25 \pm 4063.97$ & $13,864.75 \pm 3824.33$ & 0.015 & $15,247.8 \pm 3162.14$ & $12,052.2 \pm 730.34$ & $<0.0001$ \\
\hline MEP & $3120.10 \pm 1725.95$ & $3850.95 \pm 1487.32$ & 0.046 & $3599.35 \pm 1122.42$ & $4116.0 \pm 1334.62$ & 0.151 \\
\hline HEP & $379.35 \pm 280.94$ & $645.50 \pm 373.59$ & $<0.0001$ & $541.20 \pm 350.25$ & $856.3 \pm 581.03$ & 0.004 \\
\hline LEPs & $7071.65 \pm 1754.22$ & $6961.1 \pm 2236.66$ & 0.725 & $7509.45 \pm 1852.70$ & $6920.20 \pm 2118.53$ & 0.148 \\
\hline LEPi & $7007.65 \pm 2150.80$ & $6737.15 \pm 2205.91$ & 0.480 & $6891.85 \pm 1818.86$ & $5778.10 \pm 2193.29$ & 0.010 \\
\hline LEPs/LEPi & $1.035 \pm 0.262$ & $1.096 \pm 0.300$ & 0.200 & I. $1499 \pm 0.384$ & $1.4227 \pm 0.820$ & 0.026 \\
\hline
\end{tabular}

aging therapy has less effective regenerative changes in this age interval. These regenerative changes could be amplified though by the use of products able to interfere with the characteristic aging mechanisms for this specific age group.

At the same time, concomitantly with the change in dermal thickness, the number of LEP decreased in the lower dermis, but not in the upper dermis. The LEPs/LEPi ratio also increased significantly after therapy. The decrease in the number of LEP in the lower dermis is proportional to the significant increase of MEP and HEP that quantify protein neosynthesis, as well as cytoarchitectural reorganizations of the extracellular matrix.

According to the literature, the cutaneous imaging parameters that can be considered as objective in the evaluation of the cutaneous aging/regeneration process are the following:

- LEP quantifies the degree of cutaneous hydration, inflammatory processes, solar elastosis, collagen degeneration.

- MEP and HEP quantify the structures of collagen and elastin fibers and microfibrils.

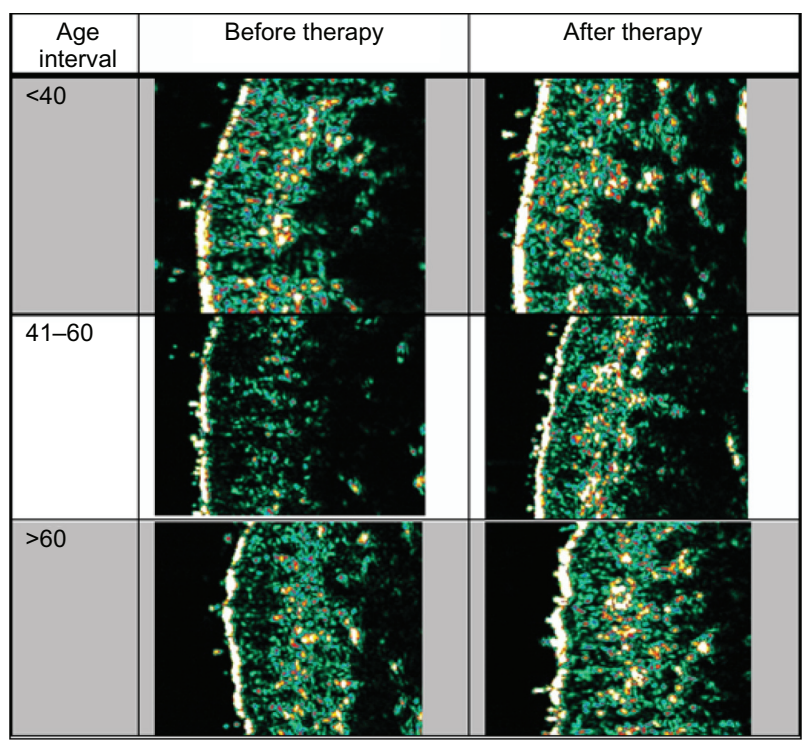

Figure 3 Ultrasonographic skin aspect before and after topical therapy, on different age groups.
- The LEPs/LEPi ratio allows an appreciation of the density and integrity of the extracellular matrix, both from the upper and lower dermis, which vary according to age, UV-ray exposure, and therapy. ${ }^{16}$

The data obtained from the study shows important ultrasonographic changes at the cutaneous level after anti-aging therapy. Flavonoids have a complex action at the dermal level, interfering with several mechanisms involved in the senescence process. They act at the level of fibrocytes, on specific receptors, turning inactive mature cells into young, metabolically active ones. ${ }^{19}$

Also, studies performed in order to assess the phytooestrogen activity of Viniferol (evaluation of gene expression, coding for typical differentiation proteins) have shown that it influences gene expression and cell proliferation both at the epidermal and dermal levels. Therefore Viniferol decreases gene expression in Loricrine, Fillagrin, and Keratin 11, and increases gene expression in Calgranulin B. ${ }^{20}$

The synthesis of the protein structures is initiated at the intracellular level. The trophocollagen molecules, elastin, and the glycosaminoglycans are extracellularly assembled into microfibrils, fibers, or proteoglycans. Depending on the biochemical structure, the level of organization, architectural orientation, and quantity, the proteins show a certain cutaneous echogenity degree. The low echogenity pixels that quantify the hydration degree of the extracellular matrix especially in the lower dermis, are replaced by medium and high echogenity pixels, quantifying protein synthesis. The MEP codify the presence of elastin and collagen microfibrils. Once these are assembled into mature fibers, the local echogenicity increases, and are expressed as hyperechogenic areas (HEP). The increase of the LEPs/LEPi ratio quantifies the replacement of the hypoechogenic pixels from the lower dermis with medium and high echogenic pixels as a result of protein neosynthesis. Type I collagen, that is predominant at the dermal level (punctiform hyperechogenic pixels) is organized in fibers, visible as hyperechogenic bands, having a parallel 
display in the lower dermis. These hyperechogenic bands, visible especially on photoprotected sites, represent an ultrasonographic marker of the intrinsic aging process. $^{21}$

The collagen-elastin protein structure, in different organization stages, is quantified by pixels of different amplitude. The post-therapeutic changes, visible on the sonograms as an increase of medium and high echogenic pixels, are suggestive of increased protein synthesis, the assembly of molecules to filaments, fibrils, and eventually, collagen fibers. According to our observations, there is a direct relationship between the genetically determined cutaneous phototype, the structure and repartition of echogenic proteins (collagen, elastin) and the different response to anti-aging therapies. ${ }^{22}$

If we consider the significant changes of the ultrasonographic parameters after anti-aging therapy depending on the phototype of the subject, a significant increase of the LEPs/ LEPi ratio is present in the subjects in phototype II class, but not class III. This observation would justify the correlation of the anti-aging therapy with the cutaneous phototype. Further studies are necessary to confirm the different reactivity of the phototype classes to local therapies.

The synthesis process of fibrillary proteins depends on several factors, involving complex, targeted, and personalized therapies. According to the "wear and tear" theory, throughout life, intrinsic and extrinsic mechanisms associated to the senescence process reduce the regenerative capacity of cells, leading to post-translational changes at the level of intracellular and extracellular proteins, such as oxidation and nonenzymatic glycation.

Flavonoids, through complex mechanisms, interfere with the reactions involved in the senescence process, and induce the synthesis of the extracellular matrix. According to our data, Viniferol-based products are more efficient in the 40-60 years age group, characterized by complex biological changes at the cutaneous level. Viniferol shows real and important anti-aging properties, since it interferes concomitantly with the genetic, oxidative, immunologic, and metabolic mechanisms that are involved in the cutaneous aging process. ${ }^{23}$

Prophylaxis of the aging process should start before the age of 40 years, preferably in the critical age group (20-40 years), which is characterized by important changes at the tissular, cellular, and molecular levels. ${ }^{24}$ The optimization of anti-aging therapy, according to special studies, requires targeted, personalized therapies, adapted to the hormonal, genetic, oxidative, immunologic, and metabolic status of the subject, and is capable of interfering with deficient mechanisms in certain age groups.
High-frequency ultrasound can be used as a modern, noninvasive, histological tool for the assessment of various anti-aging therapies, with applications in the fields of pharmacology and dermato-cosmetology.

\section{Conclusions}

High-frequency ultrasound is a modern, noninvasive method that allows the assessment of the efficacy of anti-aging therapies. After topical flavonoid-based therapy, a significant increase of the epidermis and dermis was noticed, especially in the 40-60 years age group. Concomitantly, other cutaneous changes take place: decrease in the number of LEP and increase in the MEP and HEP. The cutaneous changes are more marked in subjects belonging to phototype class II. Anti-aging prophylaxis should be initiated in the 20-40 years critical age group, involving personalized therapies also adapted to the phototype.

\section{Acknowledgments}

The authors gratefully acknowledge Cortex Technology for allowing the use of the ultrasound equipment and for their assistance with the application of the Dermascan to this project. This study is part of the research grant CNCSIS ID 2624.

\section{Disclosure}

The authors report no conflicts of interest in this work.

\section{References}

1. Seidenari S, Pagnoni A, Di Nando A, Giannetti A. Echographic evaluation with image analysis of normal skin: variations according to age and sex. Skin Pharmacol. 1994;7(4):201-209.

2. Gniadecka M. Effects of aging on dermal echogenicity. Skin Res Technol. 2001;7:204-207.

3. Schmid-Wendtner MH, Burgdorf W. Ultrasound scanning in dermatology. Arch Dermatol. 2005;141:217-224.

4. Lasagni C, Seidenari S. Echographic assessment of age dependant variations of skin thickness: a study on 162 subjects. Skin Res Technol. 1995;1:81-85.

5. Tsukhara K, Takema Y, Moriwaki S, Fujimura T, Kitahara T, Immokava G. Age related alterations of echogenicity in Japanese skin. Dermatology. 2000;200(4):303-307.

6. Pellacani G, Seidenari S. Variations in facial skin thickness and echogenicity with site and age. Acta Derm Venereol. 1999;79(5):366-369.

7. De Rigal J, Escoffler C, Querleux B, Faivre B, Agache P. Assessment of the human skin by in vivo ultrasonic imaging. I Invest Dermatol. 1989;93(5):621-625.

8. Sator PGT, Schmidt JB, Honigsmann H. Objective assessment of photoaging effects using high-frequency ultrasound in PUVA treatment psoriasis patients. Br J Dermatol. 2002;147:291-298.

9. Béguin A. A novel micronutrient supplement in skin aging: a randomized placebo-controlled double-blind study. J Cosmet Dermatol. 2005;4:277-284.

10. Sandby-Møller J, Wulf HC. Ultrasonographic subepidermal lowechogenic band, dependence of age and body site. Skin Res Technol. 2004;10(1):57-63. 
11. Gniadecka M, Gniadecki R, Serup J, Søndergaard J. Ultrasound structure and digital image analysis of the subepidermal low echogenic band in aged human skin: diurnal changes and interindividual variability. J Invest Dermatol. 1994;102:362-365.

12. Bonina F, Lanza M, Montenegro L, et al. Flavonoids as potential protective agents against photo-oxidative skin damage. Int J Pharm. 1996;145(1-2):87-94.

13. Kawada K. Risk and preventive factors for skin phototype. J Dermatol Sci. 2000;23 Suppl 1:S27-S29.

14. Moldovan M, Crisan M, Vornicescu Pop D, Mos E. Moisturising cream for dry skin and preparation protocol. OSIM deposit, nr. A00951, 2008.

15. Tsai SH, Lin-Shiau SY, Lin JK. Suppression of nitric oxide synthase and the down-regulation of the activation of NFkappaB in macrophages by resveratrol. Br J Pharmacol. 1999;126(3):673-680.

16. Man RYK, Leung SWS, Leung GPH. Effect of flavonoids on cell proliferation: comparison with estrogen. FASEB J. 2008;22:718.4.

17. Stipcevic T, Piljac J, Vanden Berghe D. Effect of different flavonoids on collagen synthesis in human fibroblasts. Plant Foods Hum Nutr. 2006;61(1):29-34.
18. Branchet MC, Boisnic S, Frances C, Robert AM. Skin thickness changes in normal aging skin. Gerontology. 1990;36(1):28-35.

19. Bastianetto S, Dumont Y, Duranton A, Vercauteren F, Breton L, Quirion R. Protective action of resveratrol in human skin: possible involvement of specific receptor binding sites. PLoS One. 2010;5(9):e12935.

20. Bernard FX, Pedretti N, Rosdy M, Deguercy A. Comparison of gene expression profiles in human keratinocyte mono-layer cultures, reconstructed epidermis and normal human skin. Exp Dermatol. 2002;11: 59-74.

21. Pierard-Franchimont C, Letawe C, Goffin V, Piérard GE. Skin waterholding capacity and transdermal estrogen therapy for menopause: a pilot study. Maturitas. 1995;22(2):151-154.

22. Guinot C, Malvy DJ, Ambroisine L, et al. Relative contribution of intrinsic vs extrinsic factors to skin aging as determined by a validated skin age score. Arch Dermatol. 2002;138(11):1454-1460.

23. Hollman PCH, Katan MB. Dietary flavonoids: intake, health effects and bioavailability. Food Chem Toxicol. 1999;37:937-942.

24. Crisan M, Cattani C, Badea R, et al. Modelling cutaneous senescence process. Computational science and its applications. ICCSA. 2010;6017 215-224.
Clinical, Cosmetic and Investigational Dermatology

\section{Publish your work in this journal}

Clinical, Cosmetic and Investigational Dermatology is an international, peer-reviewed, open access, online journal that focuses on the latest clinical and experimental research in all aspects of skin disease and cosmetic interventions. All areas of dermatology will be covered; contributions will be welcomed from all clinicians and

\section{Dovepress}

basic science researchers globally. This journal is indexed on CAS The manuscript management system is completely online and includes a very quick and fair peer-review system, which is all easy to use. Visit http://www.dovepress.com/testimonials.php to read real quotes from published authors. 